\title{
On the near-critical behavior of cavitation in elastic plane membranes
}

\author{
Y.N. Geng ${ }^{\mathrm{a}}$, Z.X. Cai ${ }^{\mathrm{a}, *}$, Y.B. Fu ${ }^{\mathrm{b}, *}$ \\ ${ }^{a}$ Department of Mechanics, Tianjin University, Tianjin 300072, China \\ ${ }^{b}$ Department of Mathematics, Keele University, Staffordshire ST5 5BG, UK
}

\begin{abstract}
Material cavitation under tensile loading is often studied by assuming the pre-existence of a small void. In this case the void would initially grow but without significant change in its size, and cavitation is said to take place if this slow growth is followed by rapid growth at higher load values. In the limit when the original void radius $\delta$ tends to zero, there will be no growth until a load or stretch measure, $\lambda$ say, reaches a well-defined critical value $\lambda_{\text {cr }}$ at which a cavity appears suddenly. In this paper we study the near-critical asymptotic behavior of cavitation in plane membranes when $\delta$ is not zero but small, and show that the near-critical behavior is governed by a scaling law in the form $\lambda-\lambda_{\text {cr }}=C(\delta / L)^{m}$, where $L$ is the undeformed outer radius of the plane membrane, and $C$ and $m$ are non-dimensional constants. The positive power $m$ in general depends on the material model used, but for the three classes of material models considered, it happens to be equal to $2(1+\nu) /(3+\nu)$ in each case, where $\nu$ is Poisson's ratio for infinitesimal deformations. If a pre-existing void is viewed as an imperfection, then this scaling law describes the imperfection sensitivity of cavitation: it states that in the presence of imperfections significant void growth would occur when $\lambda$ were increased to within an order $(\delta / L)^{m}$ interval around $\lambda_{\mathrm{cr}}$.
\end{abstract}

Keywords: Cavitation, imperfection sensitivity, void growth, membrane

\section{Introduction}

In some tension experiments on rubber materials [1-4], it is shown that internal micro-voids could nucleate suddenly (known as cavitation) under certain critical loading conditions. With continued loading a series of cavities may grow, coalesce, and eventually form large enclosed cracks. Thus, cavitation may signal the onset of material failure.

Cavitation in rubber materials was first demonstrated experimentally by Gent and Lindley [1] who also provided a theoretical explanation, but it was not until after Ball [5] had formulated it as a rigorous bifurcation problem that an explosive growth of interest followed. In its simplest form, namely an isotropic sphere with a small void at its center that is subjected to a hydrostatic

\footnotetext{
*Corresponding authors

Email address: zxcai@tju.edu.cn (Z.X. Cai), y.fu@keele.ac.uk(Y.B.Fu)
} 
tension on its outer surface, the cavitation problem consists of solving a two-point boundary value problem involving a single nonlinear second-order ordinary differential equation. The critical tension can be obtained by taking two limits one after the other: firstly the undeformed cavity radius tending to zero, and then the deformed cavity radius tending to zero. This has been demonstrated by Horgan and Abeyaratne [6], and is justified by the rigorous results of Sivaloganathan et al. [7]. When the material is incompressible, the radially axisymmetric deformation can be determined to within an arbitrary constant irrespective of the form of the strain-energy function, and determination of the critical tension and post-buckling deformation is then reduced to the evaluation of an integral. When the material is compressible, the two-point boundary value problem can be solved by a shooting procedure in the most general case (see, e.g., [8]), but many studies have focused on finding closed-form solutions for specific material models (see, e.g., [9-12]). There also exists a large body of literature concerned with the effects of anisotropy, material inhomogeneity, surface tension, and plastic behavior; see, e.g., [13-19]. We refer to [20] and [21] for a comprehensive review of the literature. An interesting result that deserves special mentioning is that whereas cavitation in a homogeneous isotropic solid sphere is a supercritical bifurcation, cavitation may change into a subcritical bifurcation when material inhomogeneity or anisotropy is taken into account.

Because of its relevance in a wide range of applications, cavitation in solids is still a topic of active research. Cristiano et al. [22] and Hamdi et al. [23] have recently conducted further experiments in order to understand the connection between cavitation and material fracture. Dorfmann and Burtscher [24] and Kumar et al. [25] suggested that cavitation was the primary cause of irreversible stress softening in seismic bearings; the same opinion was expressed for metal materials [26-28]. Increased attention has also been paid to cavitation associated with general geometries and loading conditions, and its numerical computations [29-33]. More closely related to the current study is the growing interest in cavitation in soft and biological materials. For example, David and Humphrey [34] studied the stress and strain concentration due to the introduction of a circular hole in an anisotropic bio-tissue, Merodio and Saccomandi [35] studied the effect of fibre-reinforcement in the radial direction, McMahon et al. [36] and Pence and Tsai [37-38] investigated cavitation due to growth and swelling, respectively, and Volokh [39] suggested that cavitation instability could be a rational indicator of aneurysm rupture.

This paper is concerned with the asymptotic properties of cavitation solutions, an aspect of the cavitation problem that seems to have been under-studied. Horgan and Abeyaratne [6] considered cavitation associated with a Blatz-Ko material, and derived two asymptotic expressions for the deformed void radius $r(\delta)$ valid for $\lambda<\lambda_{c r}$ and $\lambda>\lambda_{c r}$, respectively, where $\delta$ is the undeformed void radius, $\lambda$ is the azimuthal stretch imposed at the outer surface and $\lambda_{c r}$ its critical value. It was shown that when $\lambda<\lambda_{c r}$, the deformed radius $r(\delta)$ is of order $\delta$, whereas when $\lambda>\lambda_{c r}$, the leading order term in $r(\delta)$ is independent of $\delta$, indicating that in this parameter regime the azimuthal stretch $r(\delta) / \delta$ would tend to infinity as $\delta \rightarrow 0$. We note, however, that these expansions would break down in the limit $\lambda \rightarrow \lambda_{c r}$, which is the parameter regime to be 
examined in the current paper. Some asymptotic results have been derived by Negrón-Marrero and Sivaloganathan [40] to aid their numerical calculations. In particular, they showed that even for a general strain-energy function the deformed cavity radius is given by $r(0)=C\left(\lambda-\lambda_{\mathrm{cr}}\right)^{1 / n}$ to leading order as $\lambda$ approaches $\lambda_{\text {cr }}$, where $C$ is a positive constant and $n$ is the dimension of the cavitation problem.

It is well-known that although cavitation is a bifurcation phenomenon, it cannot be studied using the traditional methods of linear and weakly nonlinear bifurcation analysis - it is an intrinsically nonlinear problem. Despite this difference, the bifurcation diagram, showing the dependence of cavitation size on the applied tensile pressure, is nonetheless of the same shape as that for more traditional buckling problems such as the pitch-fork bifurcation associated with Euler buckling. A pre-existing void can be viewed as an imperfection, and the associated imperfect bifurcation diagram can be viewed as an "unfolding" of the perfect bifurcation diagram, in exactly the same manner as in Euler buckling; see, e.g., Figure 1 in [6], or the three figures in the current paper. In the case of Euler buckling, a simple near-critical analysis would yield an amplitude equation of the form

$$
\left(p-p_{\mathrm{cr}}\right) A+c_{1} A^{3}+c_{2} \delta=0,
$$

where $A$ is a measure of the unscaled buckling amplitude (e.g. the maximum deflection), $p$ is the compressive load and $p_{\text {cr }}$ its critical value, $c_{1}$ and $c_{2}$ are constants, and $\delta$ denotes the amplitude of the imperfection. According to this amplitude equation, when $p$ is much smaller than $p_{\text {cr }}$ so that $p-p_{\text {cr }}$ is finite, dominant balance is between the first and third terms in (1), which yields $A \sim \delta$, where " $\sim$ " means "is of the same order as". On the other hand, when $p-p_{\text {cr }}$ is sufficiently small so that all the three terms in (1) are of the same order, we have

$$
A \sim \delta^{1 / 3}, \quad \text { and } p-p_{\text {cr }} \sim \delta^{2 / 3}
$$

which are the scalings of most interest in the assessment of structural integrity. The final parameter regime of interest is when $p-p_{\text {cr }}$ is much larger than $\delta^{2 / 3}$, in which case dominant balance is between the first two terms, the effect of imperfection is not felt to leading order, and so the bifurcation curve tends to its counterpart in the absence of imperfections.

Thus, an amplitude equation such as (1) serves to capture the near-critical behavior, and it is well-known that the solution given by (1) gives a very good approximation to the exact solution around $p=p_{\text {cr }}$ even when $\delta$ is only moderately small. The main objective of the current study is to demonstrate that a near-critical amplitude equation analogous to equation (1) can also be derived for the cavitation problem. We shall consider three material models for which the cavitation solution can be obtained in closed-form.

The rest of this paper is organized as follows. After formulating the cavitation problem in the next section, we present the above-mentioned asymptotic analysis for three classes of material models in the subsequent sections. In the final section we reflect on our main results and conclude the paper with some additional comments. 


\section{Problem description}

We consider a uniform circular membrane containing a pre-existing circular hole with radius $\delta$ at its center, and the membrane is subjected to radial tension. The undeformed configuration occupies the region $D_{0}=\{(R, \Theta) \mid \delta \leq R \leq 1,0 \leq \Theta \leq 2 \pi\}$ in terms of plane polar coordinates, where the outer radius of the membrane has been taken to be unity, corresponding to the fact that we are using the actual radius as the unit of lengths. We assume that the resulting axisymmetric deformation is given by

$$
r=r(R), \quad \theta=\Theta
$$

where $r$ and $\theta$ are the plane polar coordinates in the current configuration, and the function $r(R)$ is to be determined. The associated principal stretches of the deformation are then given by

$$
\lambda_{r}=\frac{\mathrm{d} r}{\mathrm{~d} R}, \quad \lambda_{\theta}=\frac{r}{R}
$$

In the subsequent analysis, the subscripts $r$ and $\theta$ are interchangeable with 1 and 2 , respectively. We shall denote the azimuthal stretch at the outer boundary $R=1$ by $\lambda$, that is $r(1)=\lambda$, and take $\lambda$ as the control parameter in the subsequent bifurcation analysis. The deformed configuration then occupies the region $D=\{(r, \theta) \mid r(\delta) \leq r \leq \lambda, 0 \leq \theta \leq 2 \pi\}$.

In the absence of body forces, the only equilibrium equation that is not satisfied automatically can be written as

$$
\frac{\mathrm{d} \sigma_{r}}{\mathrm{~d} R}+\frac{\mathrm{d} r}{\mathrm{~d} R} \frac{\sigma_{r}-\sigma_{\theta}}{r}=0
$$

where $\sigma_{r}$ and $\sigma_{\theta}$ are the principal Cauchy stress components (measured per unit length in the deformed configuration). When the membrane is viewed as a two-dimensional elastic continuum with strain energy function $W\left(\lambda_{r}, \lambda_{\theta}\right)$ (measured per unit area in the undeformed configuration), the Cauchy stresses are given by

$$
\sigma_{r}=\frac{1}{\lambda_{\theta}} \frac{\partial W}{\partial \lambda_{r}}, \quad \sigma_{\theta}=\frac{1}{\lambda_{r}} \frac{\partial W}{\partial \lambda_{\theta}}
$$

Equation (4) is to be solved subject to the displacement boundary condition

$$
r(1)=\lambda,
$$

and the traction-free boundary condition

$$
\sigma_{r}(\delta)=0
$$

If the above boundary value problem in the limit $\delta \rightarrow 0$ has a non-trivial solution with $r(0)>0$ for some $\lambda$, cavitation is said to occur. The above cavitation problem was solved in a series of papers by Haughton $[8,41,42]$. His approach was to start with a 3D strain-energy function and obtain the reduced 2D strain-energy function by using the membrane assumption $\sigma_{3}=0$ to 
eliminate the principal stretch $\lambda_{3}$ in the thickness direction. In particular, he showed that for the class of strain-energy function given by

$$
W=\sum_{r=1}^{n} \mu_{r}\left(\lambda_{1}^{\alpha_{r}}+\lambda_{2}^{\alpha_{r}}+\lambda_{3}^{\alpha_{r}}-3\right) / \alpha_{r}
$$

where $\alpha_{1}<\alpha_{2}<\cdots<\alpha_{n}$, sufficient conditions for non-existence of cavitation are $\alpha_{1}>1$ or

$$
\alpha_{1}<-1 \text { and } 2 \alpha_{n}>3-\alpha_{1}
$$

but the class of strain-energy function given by

$$
W=\frac{\mu}{\alpha}\left(\lambda_{1}^{-\alpha}+\lambda_{2}^{-\alpha}+\lambda_{3}^{-\alpha}-3+\alpha\left(\lambda_{1} \lambda_{2} \lambda_{3}-1\right)\right)
$$

supports cavitation for all $\alpha>0$. In contrast, Steigmann [43] suggested the use of a direct theory, in which the membrane is regarded as a two-dimensional elastic continuum endowed with a strain energy $W$, measured per unit area of the reference surface. He found an explicit cavitation solution for a class of such strain energy given by

$$
W=2 G\left(\lambda_{1}+\lambda_{2}+f\left(\lambda_{1} \lambda_{2}\right)\right)
$$

where $G$ denotes the ground-state shear modulus times the undeformed membrane thickness, and $\lambda_{1}, \lambda_{2}$ are the two in-plane stretches. In this spirit, the explicit (plane-strain) cavitation solution found by Horgan and Abeyaratne[6] for

$$
W=\frac{\mu}{2}\left(\lambda_{1}^{-2}+\lambda_{2}^{-2}+2 \lambda_{1} \lambda_{2}-4\right),
$$

is also valid for plane membranes if we replace $\mu$ by $G$. More recently, Shang and Cheng [44] found an explicit cavitation solution for a plane membrane that is described by the Cauchy elastic model

$$
\sigma_{r}=\frac{E}{1-\nu^{2}}\left(\ln \frac{\mathrm{d} r}{\mathrm{~d} R}+\nu \ln \frac{r}{R}\right), \quad \sigma_{\theta}=\frac{E}{1-\nu^{2}}\left(\nu \ln \frac{\mathrm{d} r}{\mathrm{~d} R}+\ln \frac{r}{R}\right),
$$

where $E$ is Young's modulus and $\nu$ is Poisson's ratio. In the next three sections, we shall examine the near-critical structure of the cavitation solution corresponding to the three material models (8)- (10).

\section{Asymptotic results for the Cauchy elastic material model}

We first consider the material model (10). The cavitation problem for this material model has been solved explicitly by Shang and Cheng [44] for the case when $\delta=0$. We now adapt their results to write down the solution when $\delta$ is non-zero.

An explicit solution is possible in this case because under the variable transformation

$$
R=R(t), \quad r=\hat{r}(t), \quad t=\frac{\lambda_{r}}{\lambda_{\theta}}=\frac{R}{r} \frac{d r}{d R}
$$


eqs.(3) and (4) can be transformed into the following system of first-order linear ordinary differential equations for $R(t)$ and $\hat{r}(t)$ :

$$
\frac{\mathrm{d} R(t)}{\mathrm{d} t}=\frac{R(t)}{t(1-t) \Psi(t)}, \quad \frac{\mathrm{d} \hat{r}(t)}{\mathrm{d} t}=\frac{\hat{r}(t)}{(1-t) \Psi(t)},
$$

where $\Psi(t)=(1+\nu)-(1-\nu) t \ln t /(1-t)$, and $1+\nu=\Psi(0) \leq \Psi(t) \leq \Psi(1)=2$.

Also, the boundary condition (6) can be rewritten as

$$
R\left(t_{0}\right)=1, \quad \hat{r}\left(t_{0}\right)=\lambda,
$$

where $t=t_{0}$ corresponds to the outer boundary $R=1$.

The solution can then be obtained by elementary integration as

$$
\begin{aligned}
R(t) & =\left(\frac{t}{t_{0}}\right)^{1 /(1+\nu)} \sqrt{\frac{1-t_{0}}{1-t}} \exp \left[-\int_{t}^{t_{0}} W(s) \mathrm{d} s\right], \\
\hat{r}(t) & =\lambda \sqrt{\frac{1-t_{0}}{1-t}} \exp \left[-\int_{t}^{t_{0}} V(s) \mathrm{d} s\right], \quad\left(0 \leq t \leq t_{0} \leq 1\right),
\end{aligned}
$$

where the two known functions $W$ and $V$ are define by

$$
W(t)=V(t)+F(t), \quad V(t)=\frac{1 / \Psi(t)-1 / 2}{1-t}>0, \quad F(t)=\frac{1 / \Psi(t)-1 /(1+\nu)}{t}<0 .
$$

Substituting (14) and (15) into (10) yields the following expressions for the stresses:

$$
\begin{aligned}
\sigma_{r}(t) & =\frac{E}{1-\nu^{2}}\left[(1+\nu) \ln \lambda+\ln t_{0}+(1+\nu) \int_{t}^{t_{0}} F(s) \mathrm{d} s\right], \\
\sigma_{\theta}(t) & =\sigma_{r}(t)-\frac{E}{1+\nu} \ln t .
\end{aligned}
$$

The inner boundary condition (7) can be rewritten as

$$
R\left(t_{1}\right)=\delta, \quad \sigma_{r}\left(t_{1}\right)=0
$$

which yield

$$
\begin{gathered}
\delta=\left(\frac{t_{1}}{t_{0}}\right)^{1 /(1+\nu)} \sqrt{\frac{1-t_{0}}{1-t_{1}}} \exp \left[-\int_{t_{1}}^{t_{0}} W(s) \mathrm{d} s\right], \\
\lambda=t_{0}^{-\frac{1}{1+\nu}} \exp \left[-\int_{t_{1}}^{t_{0}} F(s) \mathrm{d} s\right], \quad\left(0 \leq t_{1} \leq t_{0} \leq 1\right) .
\end{gathered}
$$

When the initial cavity radius $\delta$ and stretch $\lambda$ are given, we can solve the simultaneous equations (19) and (20) to obtain $t_{1}$ and $t_{0}$. Then the displacement and stress fields in the membrane are fully determined parametrically.

Equation (19) shows that the limit $\delta \rightarrow 0$ corresponds to $t_{1} \rightarrow 0$. In this limit, cavitation occurs if $r(0)$ is non-zero, and the critical load is determined by taking the further limit $r(0) \rightarrow$ 
0 , i.e. $t_{0} \rightarrow 1$, as mentioned in the Introduction. Thus from (20) the critical stretch $\lambda_{c r}$ is given by

$$
\lambda_{c r}=\exp \left[-\int_{0}^{1} F(s) \mathrm{d} s\right] .
$$

We now use the parametric solution obtained above to derive its asymptotic behavior when both $\left|\lambda-\lambda_{\mathrm{cr}}\right|$ and $\delta$ are small. To this end, we assume that

$$
\lambda=\lambda_{c r}+C \delta^{m}, \quad t_{1} \ll 1, \quad t_{0}=1-t_{00} \delta^{n}
$$

where the positive powers $m$ and $n$, and the precise order of $t_{1}$ are to be determined by the principle of dominant balance.

On substituting (22) into (20) and taking the logarithms of both sides, we obtain

$$
\ln \left(\lambda_{c r}+C \delta^{m}\right)+\frac{1}{(1+\nu)} \ln \left(1-t_{00} \delta^{n}\right)+\int_{t_{1}}^{1-t_{00} \delta^{n}} F(s) \mathrm{d} s=0 .
$$

We observe that $F(s)$ is regular as $s \rightarrow 1$ but is singular as $s \rightarrow 0$. More precisely, we have

$$
F(s)=\frac{1-\nu}{(1+\nu)^{2}} \ln (s)+\frac{(1-\nu) s \ln s[1+\nu+(1-\nu) \ln s]}{(1+\nu)^{3}}+\cdots, \quad \text { as } s \rightarrow 0,
$$

and

$$
\int_{0}^{t_{1}} s F^{\prime}(s) d s=\frac{1-\nu}{(1+\nu)^{2}} t_{1}+\frac{1-\nu}{2(1+\nu)^{3}}\left\{\nu+2 \ln t_{1}+(1-\nu)\left(\ln t_{1}\right)^{2}\right\} t_{1}^{2}+O\left(t_{1}^{3}\right) .
$$

Thus, the integral in (23) may be evaluated with the aid of the asymptotic expansions

$$
\int_{0}^{1-t_{00} \delta^{n}} F(s) \mathrm{d} s=\int_{0}^{1} F(s) \mathrm{d} s-F(1) t_{00} \delta^{n}+O\left(\delta^{2 n}\right)
$$

and

$$
\int_{0}^{t_{1}} F(s) \mathrm{d} s=t_{1} F\left(t_{1}\right)-\int_{0}^{t_{1}} s F^{\prime}(s) \mathrm{d} s=t_{1}\left[F\left(t_{1}\right)-\frac{1-\nu}{(1+\nu)^{2}}\right]+O\left(\left(t_{1} \ln t_{1}\right)^{2}\right) .
$$

As a result, equation (23) may be approximated by

$$
\frac{C}{\lambda_{c r}} \delta^{m}-t_{1}\left[F\left(t_{1}\right)-\frac{1-\nu}{(1+\nu)^{2}}\right]-t_{00}\left[\frac{1}{1+\nu}+F(1)\right] \delta^{n}=0 .
$$

It can be seen that dominant balance is achieved by taking $n=m$ and

$$
t_{1} F\left(t_{1}\right) \sim t_{1} \ln t_{1} \sim \delta^{m}
$$

It then follows that $t_{1}$ must be of order $\delta^{m} / \ln \delta$. To simplify analysis, we may simply treat $\ln \delta$ as an $O(1)$ quantity and expand $t_{1}$ as

$$
t_{1}=t_{10} \delta^{m}+\cdots,
$$


where $t_{10}$ may be treated as an $O(1)$ constant although strictly speaking it is of order $1 / \ln \delta$. Equation (24) then reduces to

$$
\frac{C}{\lambda_{c r}}-t_{10}\left[F\left(t_{10} \delta^{m}\right)-\frac{1-\nu}{(1+\nu)^{2}}\right]-t_{00}\left[\frac{1}{1+\nu}+F(1)\right]=0 .
$$

Similarly, with the use of (22) and (25), equation (19) may be approximated to leading order by

$$
\left(t_{10}\right)^{1 /(1+\nu)} \cdot\left(t_{00}\right)^{\frac{1}{2}} \cdot \exp \left[-\int_{0}^{1} W(s) \mathrm{d} s\right] \cdot \delta^{\frac{(3+\nu) m}{2(1+\nu)}}=\delta .
$$

Dominant balance then requires that

$$
m=\frac{2(1+\nu)}{3+\nu}
$$

and as a result (27) reduces to

$$
t_{00}=K t_{10}^{-\frac{2}{1+\nu}}
$$

where

$$
K=\exp \left[2 \int_{0}^{1} W(s) \mathrm{d} s\right]
$$

From (15) we obtain the leading-order expansion

$$
r(\delta)=\hat{r}\left(t_{1}\right)=\delta^{\frac{m}{2}} \cdot t_{10}^{-\frac{1}{1+\nu}}+\cdots
$$

Finally, on eliminating $t_{10}$ from (26) and (29), we then obtain the amplitude equation

$$
\frac{\lambda-\lambda_{\mathrm{cr}}}{\lambda_{\mathrm{cr}}} r(\delta)^{1+\nu}-\frac{1}{2} K r(\delta)^{3+\nu}+\frac{1-\nu}{(1+\nu)^{2}} \delta^{1+\nu}\left[1-(1+\nu) \ln \frac{\delta}{r(\delta)}\right]=0,
$$

which can be used to compute the dependence of the deformed cavity radius $r(\delta)$ on $\lambda$. In Figure 1 we have shown this leading-order asymptotic result together with the exact solution computed numerically, for the three typical values of $\delta$ indicated in the figure. All our numerical calculations are carried out with the aid of Mathematica [45]. It is seen that the leading-order asymptotic results capture very well the near-critical behavior.

\section{Asymptotic results for the Varga material model}

We next consider the model (8) which has previously been considered by Steigmann [43] by assuming $\delta=0$; see also Haughton [42]. The assumptions $f(1)=-2, f^{\prime}(1)=-1$ are imposed to ensure that the undeformed state is energy-free and stress-free.

It can be shown that in this case the equilibrium equation may be reduced to the form

$$
\lambda_{2}^{2} \frac{d \lambda_{1}}{d \lambda_{2}}+J=0
$$




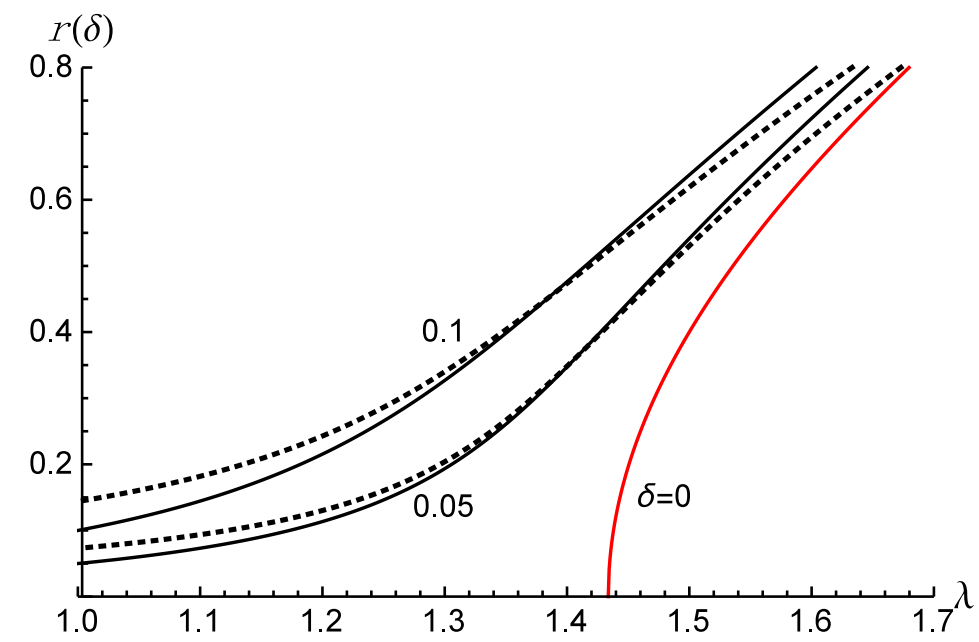

Figure 1: Variation of $r(\delta)$ with respect to $\lambda$ for the three typical values of cavity size indicated when the Cauchy elastic material model is used. Solid lines are exact results obtained by numerical calculations, and the dashed lines represent the leading-order asymptotic results.

where $J=\lambda_{1} \lambda_{2}$. It then follows on integration that $J$ must necessarily be a constant, which we denote by $J_{0}$. On the other hand, on substituting (3) into $\lambda_{1} \lambda_{2}=J_{0}$ and then integrating the resulting equation subject to the boundary condition (6), we obtain

$$
\lambda^{2}=\left(1-\delta^{2}\right) J_{0}+r^{2}(\delta) .
$$

Finally, the traction-free boundary condition (7) yields the result

$$
r(\delta)=-\frac{\delta}{f^{\prime}\left(J_{0}\right)} .
$$

In the limit $\delta \rightarrow 0, r(\delta)$ is bounded, and so we must have $f^{\prime}\left(J_{0}\right)=0$, the solution of which we denote by $J_{0}=J_{\text {cr }}$ (thus $J_{\text {cr }}$ is where $f\left(J_{0}\right)$ attains its minimum). Equation (32) then gives the amplitude equation $r^{2}(0)=\lambda^{2}-J_{\mathrm{cr}}$, which shows that the cavitation solution is only possible for $\lambda \geq \sqrt{J_{\text {cr }}}$. Hence the critical stretch is given by $\lambda_{\text {cr }}=\sqrt{J_{\text {cr }}}$, which justifies the presence of the subscript "cr"in $J_{\text {cr }}$. In our numerical example, we shall make the simplest choice

$$
f(J)=\frac{1}{2}(J-2)^{2}-\frac{5}{2},
$$

which gives $\lambda_{\text {cr }}=\sqrt{2}$.

To determine the near-critical behavior for small $\delta$, we assume that

$$
\lambda^{2}=J_{\mathrm{cr}}+\lambda_{3} \delta^{m}+\cdots, \quad J_{0}=J_{\mathrm{cr}}+J_{3} \delta^{n}+\cdots,
$$

where $m, n, \lambda_{3}, J_{3}$ are all constants. Substituting these expansions into (32) and noting that

$$
r(\delta)=-\frac{\delta}{f^{\prime}\left(J_{0}\right)} \approx-\frac{\delta}{f^{\prime \prime}\left(J_{\mathrm{cr}}\right)\left(J_{0}-J_{\mathrm{cr}}\right)},
$$


we see that dominant balance is achieved with $m=n=2 / 3$, and to leading order we have

$$
\lambda_{3}=J_{3}+\frac{1}{\left(J_{3} f_{\mathrm{cr}}^{\prime \prime}\right)^{2}}, \quad \text { where } f_{\mathrm{cr}}^{\prime \prime} \equiv f^{\prime \prime}\left(J_{\mathrm{cr}}\right)
$$

Solving (35) $)_{1,2}$ for $\lambda_{3}$ and $J_{3}$, respectively, and substituting the resulting expressions into $(37)_{1}$, we obtain the leading-order amplitude equation

$$
\left(\lambda^{2}-J_{\mathrm{cr}}\right) r(\delta)-r(\delta)^{3}+\left(f_{\mathrm{cr}}^{\prime \prime}\right)^{-1} \delta=0 \text {. }
$$

It is interesting to observe that this equation has the same structure as equation (1)!

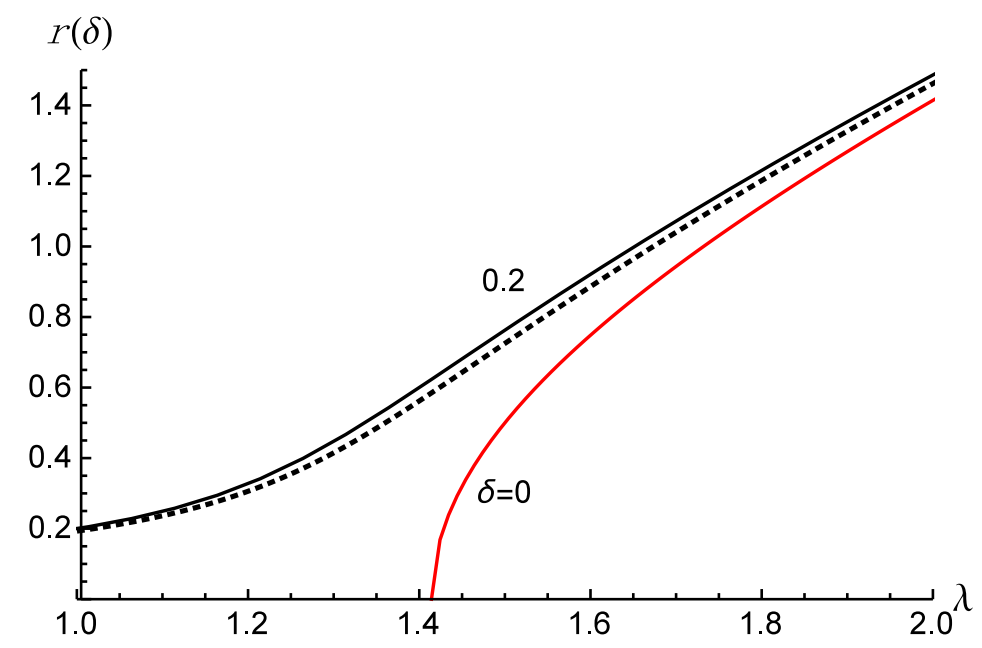

Figure 2: Variation of $r(\delta)$ with respect to $\lambda$ for the two typical values of cavity size indicated when the Varga material model is used. Solid lines are exact results obtained by numerical calculations, and the dashed lines are the leading-order asymptotic results.

In Figure 2, we have shown this leading-order asymptotic result when $\delta=0.2$ together with the corresponding exact result. It is noted that for the material model under consideration the leading-order asymptotic result provides a much better approximation than in the previous case. The asymptotic result seems to be uniformly valid for all values of $\lambda$, and it becomes graphically indistinguishable from the exact result when $\delta$ is still as large as 0.1 .

\section{Asymptotic results for the Blatz-Ko material model}

We finally consider the material model given by

$$
W\left(\lambda_{1}, \lambda_{2}\right)=\frac{G}{2}\left(\lambda_{1}^{-2}+\lambda_{2}^{-2}+2 \lambda_{1} \lambda_{2}-4\right)
$$

where the constant $G$ has the same meaning as in the previous section. Adapting the results given by Horgan and Abeyaratne[6], we first note that in this case the equilibrium equation takes the form

$$
3 R r^{3} r^{\prime \prime}(R)-r^{3} r^{\prime}(R)+R^{3}\left(r^{\prime}(R)\right)^{4}=0, \quad \delta<R<1
$$


and that in terms of the new dependent variable $t(R)$ defined by

$$
t(R)=\frac{R}{r} \frac{d r}{d R}
$$

it can be linearized:

$$
3 R t^{\prime}-t(1-t)\left(t^{2}+t+4\right)=0 .
$$

As a result the solution can be obtained in closed-form. In terms of the function

$$
h(t)=\exp \left\{\frac{6}{\sqrt{15}} \tan ^{-1}\left(\frac{2 t+1}{\sqrt{15}}\right)\right\},
$$

the critical stretch is given by

$$
\lambda_{\mathrm{cr}}=\left(\frac{27 h(1)}{8 h(0)}\right)^{1 / 8},
$$

and the deformed inner radius $r(\delta)$ is given by

$$
r(\delta)=\delta t_{b}^{-3 / 4}
$$

where the constant $t_{b}$ is related to the control parameter $\lambda$ by the equations

$$
\lambda^{8}=\frac{\left(t_{a}^{2}+t_{a}+4\right)^{3} h\left(t_{a}\right)}{t_{b}^{6}\left(t_{b}^{2}+t_{b}+4\right)^{3} h\left(t_{b}\right)}, \quad \frac{t_{a}^{6} h\left(t_{a}\right)}{\left(1-t_{a}\right)^{4}\left(t_{a}^{2}+t_{a}+4\right)}=\frac{t_{b}^{6} h\left(t_{b}\right)}{\delta^{8}\left(1-t_{b}\right)^{4}\left(t_{b}^{2}+t_{b}+4\right)} .
$$

Equation (42) $)_{2}$ defines $t_{a}$ as a function of $t_{b}$, and then (41) and (42) $)_{1}$ yield a parametric representation for the cavitation amplitude $r(\delta)$ as a function of $\lambda$.

A simple argument of dominant balance shows that the near-critical behavior is governed by the expansions

$$
\lambda=\lambda_{\mathrm{cr}}+C \delta^{4 / 5}, \quad t_{a}=1-k_{1} \delta^{4 / 5}+O\left(\delta^{8 / 5}\right), \quad t_{b}=k_{2} \delta^{4 / 5}+O\left(\delta^{8 / 5}\right),
$$

where $C, k_{1}, k_{2}$ are constants. On substituting these expansions into (42) and equating the coefficients of leading order terms, we obtain

$$
k_{1}=\frac{256 \lambda_{\mathrm{cr}}^{7} h(0)^{2} C+k_{2}\left[81 h(0) h(1)+108 h(1) h^{\prime}(0)\right]}{54 h(0)\left(9 h(1)-2 h^{\prime}(1)\right)}, \quad k_{2}=\left(\frac{2 h(1)}{3 h(0)}\right)^{1 / 6} \cdot \frac{1}{k_{1}^{2 / 3}} .
$$

Finally, on combining the expressions (41)-(44), we obtain the amplitude equation

$$
k_{3}\left(\lambda-\lambda_{\mathrm{cr}}\right) r(\delta)^{\frac{4}{3}}-k_{4} r(\delta)^{\frac{10}{3}}+k_{5} \delta^{\frac{4}{3}}=0,
$$

where

$$
\begin{gathered}
k_{3}=256 \lambda_{\mathrm{cr}}^{7} h(0)^{2}, \quad k_{4}=54 h(0)\left(9 h(1)-2 h^{\prime}(1)\right)\left(\frac{2 h(1)}{3 h(0)}\right)^{\frac{1}{4}}, \\
k_{5}=81 h(0) h(1)+108 h(1) h^{\prime}(0) .
\end{gathered}
$$

In Figure 3, we compare the leading-order result (45) with the corresponding exact result for the two values of $\delta$ indicated. Again we see that the asymptotic results provide a very good approximation for the near-critical behavior of the cavitation solutions. 


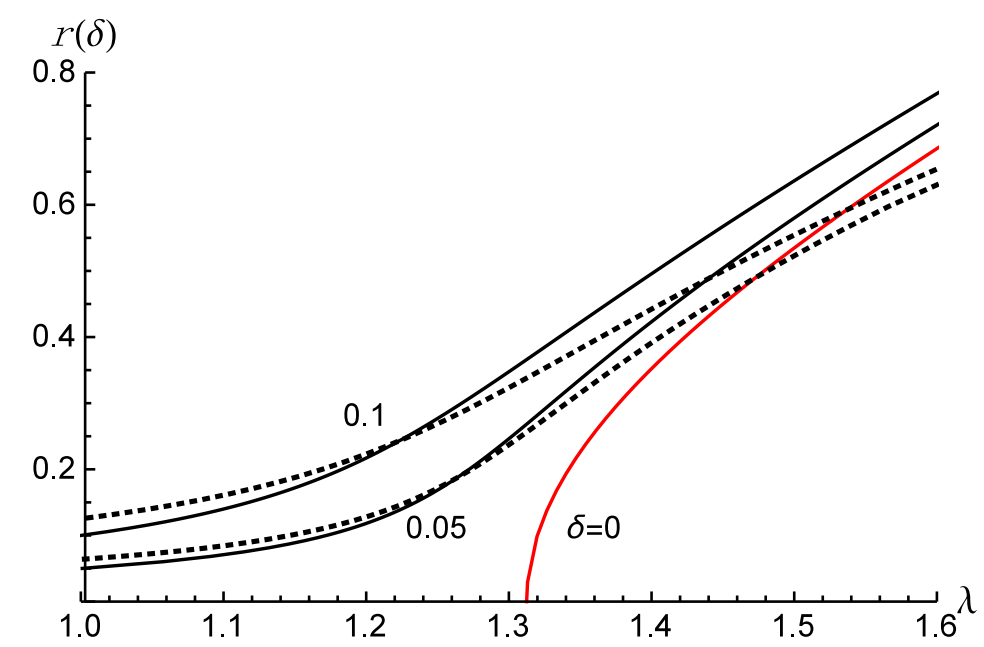

Figure 3: Variation of $r(\delta)$ with respect to $\lambda$ for the three typical values of cavity size indicated when the Blatz-Ko material model is used. Solid lines are exact results obtained by numerical calculations, and the dashed lines are the leading-order asymptotic results.

\section{Summary and discussion}

In this paper we have determined the near-critical behavior of the deformation of a stretched circular membrane with a small pre-existing void. The small void is viewed as an imperfection as in standard (non-singular) bifurcation/buckling problems. In a qualitative fracture analysis, it would be of interest to understand precisely how close the applied stretch $\lambda$ should be to $\lambda_{\text {cr }}$ when rapid void growth occurs. This question is addressed for three classes of material models. In each case the correct scalings are identified, and the leading-order asymptotic solution is shown to provide a very good approximation to the exact solution. Our amplitude equations (30), (38) and (45) have the same structure as the reference amplitude equation (1) for Euler buckling (e.g. the third term always represents the effect of imperfection), but the terms have a variety of forms (e.g. the third term in (30) is not even a power function). We note, however, that in each case when $\delta=0$ the amplitude $r(0)$ is proportional to $\sqrt{\lambda-\lambda_{\text {cr }}}$, which is consistent with the general result given by Negrón-Marrero and Sivaloganathan [40].

Although we have focused our attention on plane membranes, the same methodology can be applied to study the asymptotic near-critical structure in plane-strain or fully 3D cavitation problems provided a closed-form solution exists - no matter how complicated it may be. In particular, when the bifurcation becomes sub-critical due to material inhomogeneity or anisotropy, cavitation will become a limiting-point instability in the presence of imperfections, and the stretch maximum can be estimated with the aid of an amplitude equation similar to the ones derived in this paper. The associated stress and strain fields can also be computed if required although we have not presented such expressions for the sake of brevity. Such near-critical solutions are expected to be useful in any qualitative fracture analysis. 
In each of the three cases studied, the stretch $\lambda$ is expanded in the form

$$
\lambda=\lambda_{\mathrm{cr}}+C \delta^{m},
$$

where $m$ is equal to

$$
2(1+\nu) /(3+\nu), \quad 2 / 3, \quad 4 / 5,
$$

respectively for the three material models considered. Although in general we expect the power $m$ to depend on the material model used, we now show that the expression $2(1+\nu) /(3+\nu)$ actually reproduces the second and third numbers above when the corresponding Poisson's ratio is used.

First for the Varga material model, the Cauchy stress components are given by

$$
\sigma_{r}=2 G\left(\lambda_{\theta}^{-1}+f^{\prime}\left(\lambda_{r} \lambda_{\theta}\right)\right), \quad \sigma_{\theta}=2 G\left(\lambda_{r}^{-1}+f^{\prime}\left(\lambda_{r} \lambda_{\theta}\right)\right) .
$$

Writing

$$
\lambda_{r}=1+\varepsilon_{r}, \lambda_{\theta}=1+\varepsilon_{\theta},
$$

where $\varepsilon_{r}$ and $\varepsilon_{\theta}$ denote the strains, and linearizing the above stress-stretch equations, we obtain

$$
\sigma_{r}=2 G f^{\prime \prime}(1)\left(\varepsilon_{r}+\left(1-\frac{1}{f^{\prime \prime}(1)}\right) \varepsilon_{\theta}\right), \quad \sigma_{\theta}=2 G f^{\prime \prime}(1)\left(\varepsilon_{\theta}+\left(1-\frac{1}{f^{\prime \prime}(1)}\right) \varepsilon_{r}\right),
$$

where use has been made of the assumption $f^{\prime}(1)=-1$. It then follows that Poisson's ratio for this class of material model is given by

$$
\nu=1-\frac{1}{f^{\prime \prime}(1)} .
$$

When $f(x)$ is given by (34), we obtain $f^{\prime \prime}(1)=1$ and so $\nu=0$. The expression $2(1+\nu) /(3+\nu)$ then reduces to $2 / 3$.

For the Blatz-Ko material model, we have

$$
\sigma_{r}=G\left(1-\lambda_{r}^{-3} \lambda_{\theta}^{-1}\right), \quad \sigma_{\theta}=G\left(1-\lambda_{\theta}^{-3} \lambda_{r}^{-1}\right) .
$$

On linearizing these expressions with the aid of (48), we obtain

$$
\sigma_{r}=3 G\left(\varepsilon_{r}+\frac{1}{3} \varepsilon_{\theta}\right), \quad \sigma_{\theta}=3 G\left(\varepsilon_{\theta}+\frac{1}{3} \varepsilon_{r}\right),
$$

and so Poisson's ratio in this case is equal to $1 / 3$. The number $2(1+\nu) /(3+\nu)$ then becomes $4 / 5$, which is exactly the third number in (47).

On the other hand, the above computation for the Varga material model also suggests that $m=2(1+\nu) /(3+\nu)$ is not universal since there is no reason why $f^{\prime \prime}(1)$ can only take the unit value in that class of material model. 


\section{Acknowledgements}

This work was supported by the National Natural Science Foundation of China (Grant Nos 11672202 and 11372212), and the National Basic Research Program of China (Grant No. 2013CB035402).

\section{References}

[1] A.N. Gent, P. Lindley, Internal rupture of bonded rubber cylinders in tension, Proc. R. Soc. London A 249 (1958) 195-205.

[2] A.N. Gent, B. Park, Failure processes in elastomers at or near a rigid spherical inclusion, J. Mater. Sci. 19 (1984) 1947-1956.

[3] A. Dorfmann, Stress softening of elastomers in hydrostatic tension, Acta Mech. 165 (2003) 117-137.

[4] N.A. Hocine, A. Hamdi, M. N. Abdelaziz, P. Heuillet, F. Zaïri, Experimental and finite element investigation of void nucleation in rubber-like materials, Int. J. Solids Struct. 48 (2011) 1248-1254.

[5] J.M. Ball, Discontinuous equilibrium solutions and cavitation in nonlinear elasticity, Philos. Trans. R. Soc. London A 306 (1982) 557-610.

[6] C.O. Horgan, R. Abeyaratne, A bifurcation problem for a compressible nonlinearly elastic medium: growth of a micro-void, J. Elasticity 16 (1986) 189-200.

[7] J. Sivaloganathan, S.J. Spector, V. Tilakraj, The convergence of regularized minimizers for cavitation problems in nonlinear elasticity, SIAM J. Appl. Math. 66 (2006) 736-757.

[8] D.M. Haughton, Cavitation in compressible elastic membranes, Int. J. Eng. Sci. 28 (1990) 163-168.

[9] J.G. Murphy, Inverse radial deformations and cavitation in finite compressible elasticity, Math. Mech. Solids 8 (2003) 639-650.

[10] C.O. Horgan, On axisymmetric solutions for compressible nonlinearly elastic solids, Z. angew Math Phys. 46 (1995) 107-125.

[11] H.C. Lei, H.W. Chang, Void formation and growth in a class of compressible solids, J. Eng. Math. 30 (1996) 693-706.

[12] X.-C. Shang, C.-J. Cheng, Exact solution for cavitated bifurcation for compressible hyperelastic materials, Int. J. Eng. Sci. 39 (2001) 1101-1117. 
[13] S.S. Antman, P.V. Negrón-Marrero, The remarkable nature of radially symmetric equilibrium states of aeolotropic nonlinearly elastic bodies, J. Elasticity 18 (1987) 131-164.

[14] D.A. Polignone, C.O. Horgan, Cavitation for incompressible anisotropic nonlinearly elastic spheres, J. Elasticity 33 (1993), 27-65.

[15] X.-G. Yuan, Z.-Y. Zhu, C.-J. Cheng, study on cavitated bifurcation problems for spheres composed of hyper-elastic materials, J. Eng. Math. 51 (2005), 15-34.

[16] C.O. Horgan, T. Pence, Cavity formation at the center of a composite incompressible nonlinearly elastic sphere, J. Appl. Mech. 56 (1989) 302-308.

[17] S. Biwa, Cavitation in finite elasticity with surface energy effects, Int. J. Non-linear Mech. 41 (2006) 1084-1094.

[18] V. Tvergaard, J.W. Hutchinson, Effect of initial void shape on the occurrence of cavitation instabilities in elastoc-plastic solids, J. Appl. Mech. 60 (1993) 807-812.

[19] B. Huo, Q.-S. Zheng, Y. Huang, A note on the effect of surface energy and void size to void growth, Eur. J. Mech. A/Solids 18 (1999) 987-994.

[20] H.S. Hou, R. Abeyaratne, Cavitation in elastic and elastic-plastic solids, J. Mech. Phys. Solids. 40 (1992) 571-592.

[21] C.O. Horgan, D. Polignone, Cavitation in nonlinearly elastic solids: a review, Appl. Mech. Rev. 48 (1995) 471-485.

[22] A. Cristiano, A. Marcellan, R. Long, C.Y. Hui, J. Stolk, C. Creton, An experimental investigation of fracture by cavitation of model elastomeric networks, J. Polym. Sci. Pol. Phys. 48 (2010) 1409-1422.

[23] A. Hamdi, S. Guessasma, M.N. Abdelaziz, Fracture of elastomers by cavitation, Mater. Design 53 (2014) 497-503.

[24] A. Dorfmann, S.L. Burtscher, Aspects of cavitation damage in seismic bearings, J. Struct. Eng. 126 (2000) 573-579.

[25] M. Kumar, A.S. Whittaker, M. C. Constantinou, Experimental investigation of cavitation in elastomeric seismic isolation bearings, Eng. Struct. 101 (2015) 290-305.

[26] D. Durban, V. Birman, On the elasto-plastic stress concentration at a circular hole in an anisotropic sheet, Acta Mech. 43 (1982) 73-84.

[27] Y.-J. Chen, X.-C. Shang, Research on the thermal cavitation problem of a preexisting microvoid in a viscoelastic sphere, Mathematical Problems in Engineering 2013 (2013) Article ID 456375. 
[28] H. Jazaeri, P. J. Bouchard, M.T. Hutchings, A.A. Mamun, R.K. Heenan, Study of cavities in a creep crack growth test specimen, Procedia Struct. Integr. 2 (2016) 942-949.

[29] P.A. Kakavas, Influence of the cavitation on the stress-strain fields of compressible Blatz-Ko material at finite deformation, Int. J. Solids Struct. 39 (2002) 783-795.

[30] J. Li, D. Mayau, F. Song, A constitutive model for cavitation and cavity growth in rubberlike materials under arbitrary tri-axial loading, Int. J. Solids Struct. 44 (2007) 6080-6100.

[31] X.M. Xu, D. Henao, An efficient numerical method for cavitation in nonlinear elasticity, Math. Mod. Meth. Appl S. 21 (2011) 1733-1760.

[32] O. Lopez-Pamies, T. Nakamura, M.I. Idiart, Cavitation in elastomeric solids: II - Onset-ofcavitation surfaces for neo-Hookean materials, J. Mech. Phy. Solids 59 (2011) 1488-1505.

[33] T. Nakamura, O. Lopez-Pamies, A finite element approach to study cavitation instabilities in non-linear elastic solids under general loading conditions, Int. J. Non-Linear Mech. 47 (2012) 331-340.

[34] G. David, J. Humphrey, Redistribution of stress due to a circular hole in a nonlinear anisotropic membrane, J. Biomech. 37 (2004) 1197-1203.

[35] J. Merodio, G. Saccomandi, Remarks on cavity formation in fiber-reinforced incompressible non-linearly elastic solids, Eur. J. Mech. A-Solid. 25 (2006) 778-792.

[36] J. McMahon, A. Goriely, M. Tabor, Spontaneous cavitation in growing elastic membranes, Math. Mech. Solids 15 (2010) 57-77.

[37] T.J. Pence, H. Tsai, On the cavitation of a swollen compressible sphere in finite elasticity, 40 (2005) 307-321.

[38] T.J. Pence, H. Tsai, Swelling-induced cavitation of elastic spheres, Math. Mech. Solids 11 (2006) 527-551.

[39] K. Volokh, Cavitation instability as a trigger of aneurysm rupture, Biomech. Model. Mechan. 14 (2015) 1071-1079.

[40] P.V. Negrón-Marrero, J. Sivaloganathan, The numerical computation of the critical boundary displacement for radial cavitation, Math. Mech. Solids 14 (2009) 696-726.

[41] D.M. Haughton, On non-existence of cavitation in incompressible elastic membranes, Q. J. Mech. Appl. Math. 39 (1986) 289-296.

[42] D.M. Haughton, Elastic membranes, in Y.B. Fu and R.W. Ogden (eds) Nonlinear elasticity: theory and applications, Cambridge University Press, New York, 2001. 
[43] D. Steigmann, Cavitation in elastic membranesan example, J. Elasticity 28 (1992) 277-287.

[44] X.-C. Shang, C.-J. Cheng, Cavitation in Hookean elastic membranes, Acta Mech. Solida Sin. 15 (2002) 89-94.

[45] S. Wolfram, Mathematica: A System for Doing Mathematics by Computer, 2nd ed. AddisonWesley, California, 1991. 